\title{
Planckian energy-mass source and the dynamics of the universe: Phenomenology
}

\author{
Timashev Serge $e^{1,2}$ \\ ${ }^{1}$ National Research Nuclear University MEPhI, Moscow, Russia \\ ${ }^{2}$ Karpov Institute of Physical Chemistry, Moscow, Russia
}

\section{Email address:}

serget@mail.ru

To cite this article:

Timashev Serge. Planckian Energy-Mass Source and the Dynamics of the Universe: Phenomenology. International Journal of Astrophysics and Space Science. Vol. 2, No. 3, 2014, pp. 33-45. doi: 10.11648/j.ijass.20140203.11

\begin{abstract}
A phenomenological model of the dynamics of the Universe is suggested as an alternative of the standard model dynamics whose inadequacy is borne out by the catastrophic difference - over 40 orders of magnitude - between the cosmological constant obtained on the basis of the standard cosmological model and that derived from experimental data. The key factor in the solution of the complex of problems unresolvable within the scope of the standard theory, the problems associated with the establishment of the essence of dark energy and dark matter included, is the abandonment of the notions of the Big Bang involving the momentary generation of matter and adoption of the hypothesis for the existence of an energy-mass source of Planckian power that originated at the instant the Big Bang took place, the energy of this source being constantly generated in and evenly distributed over every element of the already originated or originating space. Possible experimental investigations aimed at gaining an insight into the issues concerning the dynamics of the Universe are discussed.
\end{abstract}

Keywords: Phenomenology, Planckian Energy-Mass Source, Dynamics of the Universe, Essence of Dark Energy and Dark Matter

\section{Introduction: Physical Vacuum in Microworld and Cosmology}

The return to the model of the dynamics of the Universe of the cosmological $\Lambda$-term, the antigravity factor introduced by Albert Einstein into the general theory of relativity (GTR) to describe a stationary, nonexpanding Universe $[1]^{1}$, was an outstanding achievement of the past decades. However, once Edwin Hubble had discovered the expanding Universe phenomenon, the need to use the $\Lambda$-term in the expanding Universe models practically vanished. It was only after the discovery in 1998 of anomalies in energy releases in Type Ia supernova explosions in distant galaxies (4-9 billion light years away) [4-7], which initiated radical changes in the views on the dynamics of the Universe, that the cosmological $\Lambda$ - term has become associated with the dark energy density $\varepsilon_{\mathrm{V}}$ :

\footnotetext{
1 The need to introduce into cosmology two types of driving force - attractive and repulsive - was recognized even in1755 by E. Kant ([2]) and in 1830 by P.Ya. Chaadaev ([3]).
}

$\varepsilon_{\mathrm{V}}=\frac{\Lambda c^{2}}{8 \pi G}$,

where $G$ and $c$ stand for the gravitational constant and velocity of light in vacuum, respectively. According to the standard cosmological model of the Universe [8-11], the quantity $\varepsilon_{\mathrm{V}}$ accounts for some $73 \%$ of the total energy density $\varepsilon_{\text {tot }}$ of the Universe. In the standard model, the dark energy density related in this way to the cosmological constant is determined by the fluctuations of the quantum chromodynamics (QCD) vacuum introduced in particle physics. It is believed that dark energy is evenly "spilled" over the Universe and satisfies the equation of state $p_{\mathrm{V}} / \varepsilon_{\mathrm{V}}=$ -1 , with the pressure $p_{\mathrm{V}}$ being negative. It is precisely this relationship, sort of implying antigravity of dark energy, that manifests itself as the factor governing the expansion of the Universe, the questions as to the physically perceptible nature of such repulsive interactions remaining open at that. Considered in the standard model is also another physically hard-to-imagine substance - dark matter - whose average energy density $\varepsilon_{\mathrm{dm}}$ accounts for some $23 \%$ of the total energy 
density of the Universe. This substance is introduced into the Friedmann equations of the dynamics of the Universe in order to remove contradictions between the apparent masses of gravitationally bound objects, as well as systems of such objects, and their observable parameters, including the structural stability of galaxies and galactic clusters in the expanding Universe. At the same time the baryonic component, whose energy density is denoted by $\varepsilon_{\mathrm{b}}$, accounts for only $4 \%$ of the total energy of the Universe. The quantity $\varepsilon_{\mathrm{b}}$ usually includes the energy density of non-baryonic components (electrons, neutrinos, electromagnetic radiations) as well.

The problems arising within the scope of the standard model, in addition to those associated with the introduction of the debatable entities - dark energy and dark matter, are accentuated because of the unsuccessful attempts to correlate the quantity $\varepsilon_{\mathrm{V}} \approx 0.66 \times 10^{-8} \mathrm{erg} / \mathrm{cm}^{3}$ observed [12] with the parameters of the QCD vacuum. The difference comes to more than 40 orders of magnitude if the characteristic energy scale is taken to be the energy scale of the QCD vacuum, $E_{\mathrm{QCD}} \sim 200 \mathrm{MeV}$ [8, 9], with the corresponding energy density being $\varepsilon_{\mathrm{QCD}}=E_{\mathrm{QCD}}^{4} /(2 \pi \hbar c)^{3}$, and would be over 120 orders of magnitude if one were focused on the vacuum of physical fields, wherein quantum and gravitational effects would manifest themselves simultaneously and the Plankian energy density $\varepsilon_{\mathrm{Pl}} \sim c^{7} / \hbar G^{2}$ would play the part of the characteristic energy scale. Such catastrophic differences, such an "orders-of-magnitude discrepancy", are considered to be a "severe trial for the entire fundamental theory" [10]. The "wanted" relations discussed in a number of works $[10,13]$, which are being suggested purely formally, without resort to comprehensible physical models linking up the energy density $\varepsilon_{\mathrm{V}}$ with the fundamental physical constants, surely cannot help solve the problem.

As will be demonstrated later in the text (see also Ref. [14]), the resolution at the phenomenological level of the above-mentioned problems arising in the standard model of the dynamics of the Universe, because of the introduction of the hypothetical entities - dark energy and dark matter - as a factor determining the expansion dynamics of the Universe, can rely on the replacement of the energy component of vacuum in the Friedmann equations by the energy density $\varepsilon^{\mathrm{e}}{ }_{\mathrm{V}}$ of the electromagnetic component of the physical vacuum - the vacuum of quantum electrodynamics (the QED vacuum). At the same time, it will also be necessary to relate to the QED vacuum a physically select frame of reference, taking it, according to [15] (see also Refs. [14, 16], to be the space of the Universe expanding in accordance with the Hubble law, so that a phenomenological relationship similar to the Friedmann equations (see elsewhere in the text) is satisfied. Established for all points of such a space - the Friedmann space-time - is a common global time $t$ reckoned from the Big Bang $(t=0)$. It will be shown later in the text that the QED vacuum can in fact act as some material substance, a present-day "aether" $[14,16]$ that can have effect on material objects and determine the observable dynamics of the entire Universe. The phenomenological basis for the pertinent estimates and the latter conclusion is provided by the well-known set of the Planck numbers that were originally introduced formally as purely numerological relationships (see Sect. 2).

Naturally various inertial systems with coordinates and time of their own can be introduced relative to such an expanding base reference system. As applied to such inertial systems, one can speak of the Lorentz invariance of various dynamical processes, but in a limited sense only. The Lorentz invariance can be violated on cosmological scales (hundreds of millions of light years and more), when the differences between the times or coordinate shifts under consideration in inertial systems exceed the characteristic cosmological values at which the nonstationarity of the dynamics of the Universe is manifest.

\section{Plankian Energy-Mass Source: Basic Relations of the Phenomenological Model of the Dynamics of the Universe}

The Planckian parameters - Planckian length, $a_{\mathrm{Pl}}$, Planckian time, $t_{\mathrm{Pl}}$, and Planckian mass, $m_{\mathrm{Pl}}-$ were introduced by Max Planck in 1899 from dimensionality considerations by combining the fundamental constants $\hbar, c$, and $G[17]$ :

$$
\begin{gathered}
a_{P l}=2^{3 / 4} \sqrt{\frac{G \hbar}{c^{3}}}=2^{1 / 2} \frac{\hbar}{m_{P l} c} \approx 2.64 \cdot 10^{-33} \mathrm{~cm}, \\
t_{P l}=\frac{a_{P l}}{c}=2^{3 / 4} \sqrt{\frac{G \hbar}{c^{5}}} \approx 0.88 \cdot 10^{-43} \mathrm{~s}, \\
m_{P l}=2^{-1 / 4} \sqrt{\frac{\hbar c}{G}} \approx 1.78 \cdot 10^{-5} \mathrm{~g} .
\end{gathered}
$$

(The choice of the numerical factors appearing in the above expressions will be explained elsewhere in the text.) The values of the length and time parameters, $a_{\mathrm{Pl}}$ and $t_{\mathrm{Pl}}$, respectively, are absolutely unattainable under conditions of physical experiment at all times [17]. Also unattainable in accelerators is the energy $m_{\mathrm{Pl}} c^{2}$ determined by the Planckian mass, though there is nothing mysterious in the mass $m_{\mathrm{Pl}}$. To unite the entire set of the Planckian numbers by imparting to them an outward feature of "unattainability", it seems natural to consider instead of the parameter $m_{\mathrm{Pl}}$ the Planckian power $w_{\mathrm{Pl}}$ that may be represented, with due regard for the numerical coefficient, in the form

$$
w_{P l}=m_{P l} c^{2} / t_{P l}=c^{5} / 2 G \approx 1.8 \times 10^{59} \mathrm{erg} / \mathrm{s} .
$$

Although the Planckian parameters have attracted attention of investigators for over 100 years now, especially since the thirties of the last century, in connection with the search for ways to naturally combine quantum mechanics 
and gravitation, the purely numerological genesis of the pertinent relations, the absence of any model arguments confirming the validity of the interest shown, left grounds for heuristic expectations only. To find such arguments, we will use Schwartzshild's solution of Einstein's equations of the GTR for the $d s^{2}$ metric of the Friedmann space-time in spherical coordinates $(r, \theta, \varphi)$ in the vicinity of a unit material object of mass $M$ (see Ref. [15]):

$$
\begin{aligned}
d s^{2}=\left(1-\frac{2 M G}{r c^{2}}\right) & c^{2} d t^{2}+2 \sqrt{\frac{2 M G}{r}} d t d r- \\
& -\left[d r^{2}+r^{2}\left(d \theta^{2}+\sin ^{2} \theta d \varphi^{2}\right)\right]
\end{aligned}
$$

It follows from expression (3) that the time component of the interval

$g_{00} \sim \psi(r)=-\left(1-r_{S c} / r\right)$ goes to zero at distances $r=$ $r_{S c}=2 G M / c^{2}$ from the object. It is well known that this result was used as an argument in favor of the existence of "black holes". If we use the different notation

$$
\psi(r)=-\left(1-w / w_{S c}\right)
$$

where the quantity $w=M c^{3} / r$ can be treated as the power of the source producing the energy $M c^{2}$ in the time $r / c$ and the parameter $w_{S c}=M c^{3} / r_{S c}=c^{5} / 2 G$ appears as the power corresponding to the Schwartzshild time $t_{\mathrm{Sc}}=r_{\mathrm{Sc}} / c$, the same logic then leads to the conclusion that the existence of an energy source with a power of $w_{\mathrm{Sc}}$ is possible. It was precisely by comparing between $w_{\mathrm{Sc}}$ (4) and $w_{\mathrm{Pl}}$ (2) and representing the Planckian length $a_{\mathrm{Pl}}=\sqrt{2} \hbar / m_{\mathrm{Pl}} c$ as the "Bohr radius" of a particle with a mass of $m_{\mathrm{Pl}}$ involved in the polarization of the QED vacuum that the numerical factors entering into expression (1) were determined. Obviously from among the above-mentioned Planckian numbers only the power $w_{\mathrm{Pl}}$, whose definition contains no Planck's constant $\hbar$, can be considered as a parameter of GTR equations containing no $\hbar$ in explicit form.

The organic unity of the parameter $w_{\mathrm{Pl}}$ with the GTR formalism is also substantiated by the fact [14] that the constantly acting hypothetical source of Planckian power, which originated at the onset of the inflatory phase of the Big Bang [18], could produce the entire energy-mass of the observable Universe during the course of its evolution. To make sure of that, let us introduce the total value $\varepsilon_{\text {tot }}$ of the average energy density, without singling out yet, as done in the standard model, individual components characterizing the respective average energy densities of the physical vacuum, "dark matter" (the physical meaning of this component will be explained later in the text), and the baryonic component. We will associate the above energy density $\varepsilon_{\text {tot }}$ with the configuration volume $V_{H}=4 \pi R_{H}^{3} / 3$ confined in the sphere of the Hubble radius $R_{H}=c / H$ (see Ref. [18]) that is sometimes defined as the "radius of the Universe". Note that according to the estimates [12], in the present epoch $H=73 \mathrm{~km} /(\mathrm{s} \cdot \mathrm{Mpc}) \approx 2.36 \times 10^{-18} \mathrm{~s}^{-1}$, so that $H^{-1} \approx 13.4$ billion years, and for the estimator of the radius of the Universe, we have $R_{\mathrm{H}}=c / H \sim 10^{28} \mathrm{~cm}$. At the same time, the average energy density of the Universe is $\varepsilon_{\text {tot }}$ $\approx 0.9 \times 10^{-8} \mathrm{erg} / \mathrm{cm}^{3}$.

We will assume that the inflatory phase of the Big Bang concluded with the origination of a hypothetical source of Planckian power, whose energy is being uniformly distributed and generated constantly in every element of the already originated and originating space, every element of the Universe's volume expanding as a result of such an energy release, though actual manifestations of this expansion can only be detected on cosmological scales. (The possible nature of the Planckian energy-mass source and the mechanism governing the transfer of its energy to the Universe is discussed in Sect. 4). In that case, one can readily estimate the amount of energy, $E_{\text {tot }}$, which the constantly acting Planckian energy-mass source could have produced during the lifetime $t$ of the Universe, if one defines this time, within the scope of the phenomenological approach being developed here, as the reciprocal of the Hubble constant, $t=H^{-1}$ (in this case, it differs by $2.2 \%$ from the 13.7 billion years' age of the Universe adopted in recent years), and assumes in addition that the power $w_{\mathrm{Pl}}$ of the Plankian energy-mass source is independent of the age of the expanding Universe. It is evident that

$$
E_{\mathrm{tot}}=w_{\mathrm{Pl}} t=\frac{c^{5}}{2 G} \cdot \frac{1}{H}=\frac{4}{3} \pi R_{H}^{3} \varepsilon_{\mathrm{tot}} .
$$

Considering that $R_{\mathrm{H}}=c / H$, we get from the above expression

$$
\varepsilon_{\text {tot }}=3 c^{2} H^{2} / 8 \pi G
$$

Expression (6) for the average density $\varepsilon_{\text {tot }}$ of the energy-mass obtained from the Planckian source of the Universe during the time $t$ of its existence/expansion in compliance with the relation $R_{\mathrm{H}}=c / H=c t$ will be used as the main relation for the Friedmann expanding base reference system being introduced, whereto the QED vacuum is believed to be related. It should be noted here that relation (5) is considered as being purely phenomenological. It is assumed that the parameters $G$ and $c$ can themselves vary with the time $t$, while the quantity $\mathcal{E}_{\text {tot }}$ does not change with time.

We take as the base physical object the three-dimensional spherical Euclidean space of the Universe that is related to the QED vacuum and varies dynamically during the global time $t$ common to all the points of the space and reckoned from the Big Bang. According to [12], the main contribution to the averaged total energy density $\varepsilon_{t o t}$ comes from the so-called dark energy which in the standard model [8-11] is identified with the energy of the physical vacuum. In contrast to [8-11], we relate to dark energy only the energy of the QED vacuum, the energy density of this component being denoted as $\varepsilon_{V}^{e}$. Further, in accordance with $[14,16]$, we 
assume that every elementary particle, every atomic nucleus polarizes the QED vacuum around itself, thus forming a "vacuum polaron" as a system bound to the QED vacuum. As shown in $[14,16]$, the energy of such a bonding between a particle of mass $m_{0}$ and the physical vacuum corresponds in magnitude to the "rest energy" of the particle in Einstein's formulation, $E_{0}=m_{0} c^{2}$. Thus, the quantity $E_{0}$ actually characterizes the binding energy of the particle-physical vacuum bond rather than the "rest energy" of the particle, so that the mass defect in nuclear processes simply characterizes the amount of energy released because of the difference in binding energy between the bonds of the original and final products to the vacuum.

We believe that it is this phenomenon - the bonding of material objects to the electromagnetic component of the physical vacuum - that is the reason for the effective "freezing" of material objects into the expanding space of the Universe, as assumed in the standard model [8-11]. It is exactly as an exhibition of such a "freezing-in" that every galaxy, every galactic cluster moves as an integral system in the course of expansion of the Universe, with the relative movements of individual stars, constellations, and nebulae within every galaxy being extremely variegated as a result of their interactions realized in accordance with the principle of least action (PLA) [19]. As in the standard model, we single out the components $\varepsilon_{\mathrm{b}}^{\mathrm{e}}$ and $\varepsilon_{\mathrm{dm}}^{\mathrm{e}}$ from the total energy density of the Universe, $\varepsilon_{\text {tot }}$. We associate with the term $\varepsilon_{\mathrm{b}}^{\mathrm{e}}$ the density of the energy binding to the QED vacuum all the mass components (both baryonic and leptonic) of the Universe in their state of rest with respect to the base space (reference system) - the Friedmann space-time. This quantity characterizing the degree of "freezing" of the resting mass components into the space of the QED vacuum is negative: $\varepsilon_{b}^{e}=-\left|\varepsilon_{b}^{e}\right|$. According to [14, 16] and Feynman's idea of the relativistic growth of mass [20], the dynamical mass and, correspondingly, the degree of "freezing-in" of cosmological objects increase in the case of their relativistic motion with respect to the Friedmann reference system, when the vacuum polarization region decreases in the travel direction, tending to zero as $u$ $\rightarrow c$. It is with this phenomenon - the growth of the degree of binding to the QED vacuum of the mass components in the case of their relativistic motion with respect to the Friedmann reference system - that we associate the energy density component $\varepsilon_{\mathrm{dm}}^{\mathrm{e}}$, which is also negative: $\boldsymbol{\varepsilon}_{d m}^{e}=-\left|\mathcal{\varepsilon}_{d m}^{e}\right|$. The subscript dm is used here to denote that the energy density so introduced is related to "dynamical mass" and not to hypothetical "dark matter", as is the case with the standard model. The definitions of $\varepsilon_{\mathrm{b}}^{\mathrm{e}}$ and $\varepsilon_{\mathrm{dm}}^{\mathrm{e}}$ being what they are, we will associate with the density of dark energy, $\varepsilon_{V}^{e}$, the difference $\varepsilon_{V}^{e}=\varepsilon_{t o t}-\varepsilon_{b}^{e}-\varepsilon_{d m}$. Naturally we retain here the values of the relative "mass" fractions $\Omega_{b}^{e}=\Omega_{b}=\left|\varepsilon_{b}^{e}\right| / \varepsilon_{\text {tot }}=0.04$ and $\Omega_{d m}^{e}=\Omega_{d m}$ $=\left|\mathcal{E}_{d m}^{e}\right| / \mathcal{E}_{\text {tot }}=0.23$ of the respective components $\mathcal{E}_{\mathrm{b}}^{\mathrm{e}}$ and $\mathcal{E}_{\mathrm{dm}}^{\mathrm{e}}$ in accordance with the data available in the literature $[12,21]$. In Sect. 3 we will adduce arguments explaining possible reasons for the sufficiently high proportion of "dark matter" (23\%) in the total energy balance of the Universe. In that case, for the relative proportion of the energy density of the QED vacuum, we get: $\Omega_{V}^{e}=$ $\varepsilon_{V}^{e} / \varepsilon_{t o t}=1.27, \Omega_{V}^{e} \neq \Omega_{V}$.

The introduction of the Planckian energy-mass source whose energy is being constantly released in every element of space is quite adequate to the representation of the dynamics of the Universe in terms of Friedmann's equations wherein the entire energy and mass are assumed to be uniformly distributed all over the Universe. It is exactly the permanent action of this energy-mass source in every element of space that can compensate for the disbalance developing constantly between the energy content of the electromagnetic component of the physical vacuum and local processes of dissipation of the energy of the QED vacuum, which incessantly sustain the states of particles and nuclei as open systems [14, 16]. The introduction of the Planckian energy-mass source permanently producing energy-mass in every elementary volume of the Universe into the model of its dynamics also agrees with the basic cosmological principle whereby every observer at one and the same instant of time finds in the Universe one and the same picture, no matter what the observation site and direction. It is with this circumstance in mind that the Friedmann equations are constructed for a simply connected closed system - the Pioncaré 3D sphere wherein all the points at rest are equivalent as to location. For such a sphere, it is traditionally assumed that the point at which the Big Bang took place is where we are now, and where all the rest of the points are found, for all the discernible points of space had been primordially found at one and the same place [17]. The recent proof by Perelman [22] of the Poincaré hypothesis that any 3D manifold is topologically equivalent to a $3 \mathrm{D}$ sphere, provided that each loop in the space can be tightened to a point, has strengthened the arguments associating our Universe with a three-dimensional sphere. If this were so, the Big Bang theory could appear more natural: if such a three-dimensional figure could be tightened to a "point", it would also be possible to extend it from the "point". Nevertheless, the absolute impossibility of imagining a three-dimensional sphere as a physical object that could have originated upon a point Bang with divergent flows of the material substance being formed, and with the complete equivalence, too, of all the points within its volume being retained during the course of its subsequent evolution, leaves grounds for doubts. The question now arises as to how the radial velocity components of the primordial substance formed from some small volume at the instant of the Big Bang are transformed into the radial components of matter scattering from every point of the volume formed at 
the subsequent instants of time. In conditions of the Planckian energy-mass source active in the Universe with a topology in the form of a three-dimensional sphere under consideration, the radial components of matter expanding from every point of the volume formed at each subsequent instant of time are being naturally compensated for, so that a medium is formed with an isotropic and homogeneous distribution of matter and pressure effective in this medium. And it is exactly this pressure, $p_{\text {eff, }}$ positive in magnitude and sustained through the action of the Planckian energy-mass source, that could do the work on increasing the volume of the Universe by "pushing apart" previously formed matter. In conditions of the Planckian energy-mass source acting in the Universe with its topology in the form of a three-dimensional sphere under consideration, the radial velocity components of matter flying away from every point of the volume formed at every subsequent instant of time are being naturally compensated for, so that a medium is formed with isotropic and homogeneous distribution of matter and the effective pressure $p_{\text {eff }}$ active therein. It is such pressure, $p_{\text {eff, }}$ positive in magnitude and maintained through the action of the Planckian energy-mass source, that could do the work on increasing the volume of the Universe by "pushing apart" previously formed matter.

We will assume that the dynamics of the expansion of the base space obeys the phenomenological Hubble relation for the rate of change, $\dot{a}$, of the scale factor $a$ :

$$
\dot{a}=H a,
$$

as well as phenomenological relation (6) that represents in integral form the total energy content of the Universe at every instant of time. Taking into consideration expression (7), relation (6) may be formally written down in the form of the 1st Friedmann equation $[8,9,18]$ for the Euclidean space, but with a different meaning of the parameters $\mathcal{E}_{\mathrm{V}}^{\mathrm{e}}$, $\mathcal{E}_{\mathrm{b}}^{\mathrm{e}}$, and $\mathcal{E}_{\mathrm{dm}}^{\mathrm{e}}$ :

$$
\left(\frac{\dot{a}}{a}\right)^{2}=\frac{8 \pi G}{3 c^{2}}\left(\varepsilon_{V}^{e}+\varepsilon_{b}^{e}+\varepsilon_{d m}^{e}\right) .
$$

To evaluate the quantity $p_{\text {eff, }}$ we consider the amount of work, $\Delta A$, done on forming fresh space by increasing the radius of the Universe, $R_{\mathrm{H}}$, by an amount of $\Delta R_{\mathrm{H}}$ in a time of $\Delta t$. Considering expression (7) and assuming that the forces responsible for the work on the expansion of the Universe, associated with the pushing apart of the already formed and matter-containing space, are governed by the QED vacuum, we have

$$
\begin{aligned}
& \Delta A=\frac{\varepsilon_{V}^{e}}{\varepsilon_{t o t}} w_{P l} \Delta t=\left.p_{\text {eff }} \Delta V_{H}\right|_{R_{H}} \\
& =\left.p_{\text {eff }} \frac{\Delta V_{H}}{\Delta R_{H}} \cdot \frac{\Delta R_{H}}{\Delta t}\right|_{R_{H}} \Delta t=p_{\text {eff }} \cdot 3 V_{H} H \Delta t
\end{aligned}
$$

and so for the quantity $p_{\text {eff }}$ we get

$$
p_{e f f}=\frac{\varepsilon_{V}^{e} c^{5}}{8 \pi \varepsilon_{t o t} R_{H}^{3} H G}=\frac{1}{3} \varepsilon_{V}^{e} .
$$

Actually expression (9) "replaces" the 2nd Friedmann equation $[8,9,18]$ in the standard model, wherefrom they usually derive the state equation relating the effective pressure $p$ averaged over all galaxies and galactic clusters to the density of the energy components of the Universe. (The pressure $p$ considered in the standard model is made negative in magnitude in order to realize "antigravity"). Estimate (9) yields $p_{\text {eff }} \approx 3.8 \times 10^{-9} \mathrm{erg} / \mathrm{cm}^{3}$, which completely agrees with the values adopted for the repulsive pressure [8-11].

It should be noted that the questions brought up when considering the system of relations (8) and (9) actually concern the mechanisms behind the action of the QED vacuum on the material objects of the Universe (the term $\varepsilon_{\mathrm{V}}^{\mathrm{e}}$ ), involving the production of the pressure $p_{\text {eff }}$ and also the possible role of the energy of binding of the material objects to the QED vacuum (the terms $\varepsilon_{\mathrm{b}}^{\mathrm{e}}$ and $\mathcal{E}_{\mathrm{dm}}^{\mathrm{e}}$ ) in the dynamics of the Universe. Such actions can be conditioned by a noticeable contribution from the quantum fluctuations of the QED vacuum to the radiation pressure (macroscopic manifestations of such effects were observed in [23]), the static Casimir effect [24, 25], and also the dynamical Casimir effect $[26,27]$ involving direct transformation of virtual photons into real photons at the boundaries of material objects traveling with relativistic velocities. We refrain here from dwelling upon the well-studied processes of nucleosynthesis at the primordial Big Bang, the subsequent incessant star formation processes, including those initiated by the energy transferred from the Planckian energy-mass source (Sect. 4), and the processes of formation of heavy elements taking place at supernova explosions [18]. Naturally such processes of production of the baryonic components of matter are accompanied with the formation of the quantum chromodynamics (QCD) vacuum characterized by the energy scales of its own [8-11], adequate energies being naturally needed to excite such vacuum, inclusive of the generation of particle-antiparticle pairs. It should be emphasized here that the "inclusion" of the Planckian energy-mass source as a factor governing the dynamics of the Universe in every elementary volume of its space is not manifest in some specific features of the dynamics, because of the going to zero, according to (3), of the contribution to the time component of the Friedmann space-time interval (see also Sect. 4). The phenomenological concepts being developed have made it possible to resolve the problems arising in the standard theory, those associated with the introduction of "dark energy" and "dark matter" included, from a unified position - with the Planckian energy-mass source introduced into the dynamics of the Universe and the base reference system with common global time used. The QED vacuum here also plays the part of the substance that unites and forms all the known types of interaction strong, electromagnetic, weak, and gravitational $[14,16]$.

In our previous works $[14,16]$, for the sake of 
convenience and uniformity of expression in dimensionless form of the fundamental strong, electromagnetic, weak, and gravitational interaction constants $-\alpha_{\mathrm{s}}, \alpha_{\mathrm{e}}, \alpha_{\mathrm{F}}$, and $\alpha_{\mathrm{g}}$, respectively, we modified the well-known Weinberg numerological relation [28] for the Planck constant $\hbar$, it being represented in the form

$$
\hbar=\frac{1}{2 \pi} G^{1 / 2} m_{Q}^{3 / 2} R_{H}^{1 / 2}
$$

with the inclusion of the energy parameter $E_{\mathrm{Q}}=m_{\mathrm{Q}} c^{2} \approx$ $209.5 \mathrm{MeV}$ characterizing the specific energy of reorganization of the physical vacuum and corresponding to an elementary quantum of action. Also introduced were the appropriate spatial scale $a_{Q}=2^{1 / 2} \hbar / m_{Q} c \approx 1.310^{-13} \mathrm{~cm}=$ 1.3 fermi - the "Bohr radius" associated with the mass $m_{\mathrm{Q}}$ - and the time scale $\tau_{\mathrm{Q}}=a_{\mathrm{Q}} / c$. In that case, the expressions presented in [16] for $\alpha_{\mathrm{g}}, \alpha_{\mathrm{F}}$, and $\alpha_{\mathrm{s}}$ assume the form

$$
\begin{gathered}
\alpha_{g}=\frac{G m_{Q}^{2}}{\hbar c}=2^{3 / 2} \pi^{2} \frac{a_{Q}}{R_{H}} \approx 2.9 \cdot 10^{-40} ; \\
\alpha_{F}=\frac{G_{F}}{a_{Q}^{2} \hbar c} \approx 0.26 \cdot 10^{-6} ; \quad \alpha_{s}=\sqrt{2} .
\end{gathered}
$$

Here $G_{\mathrm{F}}=1.436 \times 10^{-49} \mathrm{erg} \cdot \mathrm{cm}^{3} \approx 1.17 \times 10^{-5}(\hbar c)^{3} \mathrm{GeV}^{-2}$ is Fermi's four-fermion interaction constant [29].

The use of the introduced characteristic quantities $a_{\mathrm{Q}}$ and $m_{\mathrm{Q}}$ allows the Planckian numbers $a_{\mathrm{Pl}}, t_{\mathrm{Pl}}, m_{\mathrm{Pl}}$, and $w_{\mathrm{Pl}}$ written down above (see expressions (1) and (2)) to be represented in a more "compact" form:

$$
\begin{array}{r}
a_{P l}=2 \pi a_{Q}\left(\frac{a_{Q}}{R_{H}}\right)^{1 / 2}, t_{P l}=2 \pi \tau_{Q}\left(\frac{a_{Q}}{R_{H}}\right)^{1 / 2}, \\
m_{P l}=\frac{1}{2 \pi} m_{Q}\left(\frac{R_{H}}{a_{Q}}\right)^{1 / 2}, w_{P l}=\frac{1}{(2 \pi)^{2}} \frac{m_{Q} c^{2}}{\tau_{Q}} \cdot \frac{R_{H}}{a_{Q}} .
\end{array}
$$

These relations demonstrate the cosmological essence of the "smallness" of the Planckian parameters $a_{\mathrm{Pl}}$ and $t_{\mathrm{Pl}}$, as well as the cosmological scale of the quantities $m_{\mathrm{Pl}}$ and $w_{\mathrm{Pl}}$. It is exactly such representation of the Planckian numbers that can be considered as a confirmation of the validity of the more than hundred years' interest in these unique combinations of the universal constants. And it seems that it is not only Planckian numbers (1), but also the modified Weinberg representation of the Planck constant, introduced in $[14,16]$ and used as the basis for expression (10), that are the phenomenological, rather than numerological relations implying as yet unresolved mysteries of our Universe.

The relations obtained also allow one to gain an insight into the "orders-of-magnitude paradox" of the modern-day standard cosmological model [8-11] mentioned above and resolved in this work on the basis of the idea that dark energy represents the energy density of the QED vacuum, $\varepsilon_{\mathrm{V}}^{\mathrm{e}}$, which determines the cosmological constant $\Lambda=8 \pi D \varepsilon_{\mathrm{V}}{ }^{\mathrm{e}} / c^{4}$, and not the energy of the quantum chromodynamics vacuum of the standard model. Thanks to the constant action of the Planckian energy-mass source, no problems arise here with the establishment of the causes of the perpetual expansion of the Universe and no need exists to postulate "antigravity" artificially (at the cost of the "orders-of-magnitude paradox"). The relations presented below lay bare the cosmological nature of the smallness of the proportionality factors between $\varepsilon_{\mathrm{V}}{ }^{\mathrm{e}}$ and the energy densities used in [8-11] for the physical vacuum:

$$
\begin{gathered}
\varepsilon_{V}^{e}=3 \times 2^{-5 / 2} \Omega_{V}^{e} \frac{a_{Q}}{R_{H}} \varepsilon_{Q C D} \approx 0.87 \times 10^{-41} \varepsilon_{Q C D}, \\
\varepsilon_{V}^{e}=3 \times 2^{1 / 2} \pi \Omega_{V}^{e}\left(\frac{a_{Q}}{R_{H}}\right)^{3} \varepsilon_{P l} \approx 1.08 \times 10^{-121} \varepsilon_{P l} .
\end{gathered}
$$

To conclude this section, it should be emphasized that the notion expounded in this work that dark energy is essentially the energy of the QED vacuum is based, in accordance with Chernin's opinion [10], on the use in the pertinent calculations of one of the Planckian parameters, namely, the Planckian power $w_{\mathrm{Pl}}$ (expression (5)).

\section{Manifestations of the Physical Vacuum in Various Periods of Evolution of the Universe}

The Planckian energy-mass source model has not only made it possible to relate the value of one of the Planckian parameters, namely, the Planckian power, to the observable value of the density of dark energy, which is being associated with the electromagnetic component of the physical vacuum, and abandon the physically little comprehensible idea of antigraviatation in the dynamics of the Universe, but also opened the way to gain a consistent insight into a number of other cosmological problems concerned with the establishment of reasons why the evolution dynamics of the Universe differed between different epochs. To resolve such problems within the scope of general phenomenology, it will be necessary to introduce notions of the possible dependence of the world constants $G$, $\hbar$, and $c$ on the Hubble parameter $H=t^{-1}$, where $t$ is the age of the Universe, or on the appropriate dimensionless variable $h(t)=H / H_{0}$, where $H_{0}$ is the value of the Hubble parameter in our epoch. When discussing such a possibility, attention should primarily be focused on the practically negligible value (around $0.6 \times 10^{-6}$ ) of the relative change $\Delta \alpha_{\mathrm{e}} / \alpha_{\mathrm{e}}$ of the fine structure constant $\alpha_{\mathrm{e}}=e^{2} / \hbar c \approx 1 / 137$ for the regions of the Universe with red shifts $z>0.4$ [30]. The arguments adduced above in favor of the natural relation of the Planckian power to the Schwarzschild solution, as well as to the formation of the total energy content of the Universe in terms of the Friedmann equations, give grounds to treat $w_{\mathrm{Pl}}=$ $c^{5} / 2 G$ as one more, in addition to $\alpha_{e}$, combination of world constants independent of the age $H^{-1}$ of the Universe.

For the subsequent qualitative estimations, we will 
simplify still more the situation and take it that the density $\varepsilon_{\text {tot }}$ of local energy content and the elementary charge $e$ are independent of $H$. In that case, it follows from the definition of $\alpha_{\mathrm{e}}$, expression (2) for $w_{\mathrm{Pl}}$, and relations (6), (10)-(12) that

$$
\begin{aligned}
& c(t)=c_{0} \cdot h(t)^{2 / 3}, G(t)=G_{0} \cdot h(t)^{10 / 3}, \hbar(t)=\hbar_{0} \cdot h(t)^{-2 / 3}, \\
& m_{Q}(t)=m_{Q 0} \cdot h(t)^{-13 / 9}, \alpha_{g}(t)=\alpha_{g 0} \cdot h(t)^{4 / 9}
\end{aligned}
$$

where the subscript " 0 " indicates quantities corresponding to the present-day epoch. The assumptions made are adequate to a qualitative discussion of various problems facing modern cosmology, as demonstrated by the examples presented below.

\subsection{Specificities of the Dynamics of the Universe at the Early Stages of its Evolution}

One of the problems before the present-day cosmology pertains to the understanding of the reasons why the specific energy density of the most powerful energy sources originated at the early stages of the evolution of the Universe had been substantially higher (by up to a thousand times! [31]) than the corresponding quantities observed for subsequent epochs. It had been just the early stages of the evolution of the Universe, characterized by red shifts of $z \sim 1$ and more, at which quasars had originated and gamma-ray bursters had made their appearance. According to Ref. [32], largely responsible for the formation of the far-infrared and submillimeter radiation background detected today, whose total energy is comparable with the total optical radiation background, are hundreds of individual sources with $z \geq 1.2$, associated with the formation of stars and galaxies, which can be resolved with the aid of modern equipment. The existence of powerful energy sources of this type in the subsequent stages of the evolution of the Universe was not detected.

Such differences in the character of formation of stars and powerful energy sources at the early stages of the evolution of the Universe are associated in the standard model [8-11] with the relatively higher proportion of energy density accounted for in these early epochs by "ordinary" (luminous) matter, $\varepsilon_{\mathrm{b}}$, and dark matter, $\varepsilon_{\mathrm{dm}}$, compared to the density $\varepsilon_{\mathrm{V}}$ of dark energy, so that $\varepsilon_{\mathrm{V}}<\varepsilon_{\mathrm{m}}=\varepsilon_{\mathrm{dm}}+\varepsilon_{\mathrm{b}}$. Since it is assumed in this case that the energy densities $\varepsilon_{\mathrm{dm}}$ and $\varepsilon_{\mathrm{b}}$ naturally decrease with increasing volume of the Universe (all the mass components had originated at the Big Bang), while the quantity $\varepsilon_{\mathrm{V}}$ remains unchanged [8-11], the total energy density $\varepsilon_{\text {tot }}=\varepsilon_{\mathrm{m}}+\varepsilon_{\mathrm{V}}$ that had occurred at the early stages of the evolution of the Universe had to exceed the quantity $\varepsilon_{\text {tot }}$ for the later stages, thus making for intense star formation processes $[8,9]$. But how can one comprehend at that the unexpectedly rapid chemical evolution of the early universe, as indicated by the results of the chemical analysis of one of the galaxies 12.4 billion light years distant from Earth, wherein nitrogen and carbon were discovered [33]?

Within the scope of the notions being developed, with the total energy density $\varepsilon_{\text {tot }}$ remaining the same, it is natural to associate the intense star formation, as well as the possibility of acceleration of the pace of chemical evolution, at the early stages of the evolution of the Universe, characterized by high $H$ values, with the different values of the "world constants" that had obtained in those epochs, as follows from relations (15). The abnormally high specific density of formation of the most powerful energy sources at the early stages of the evolution of the Universe should be first of all associated with the higher, according to relation (15), value of the specific gravitation constant $\alpha_{g}$. It can also be assumed that the more intense formation of stars and quasi-star systems had been facilitated, according to relation (15), by the higher values of the velocity of light, $c$, and also the lower values of the Planck constant $\hbar$. Indeed, higher $c$ values allow for high velocities and kinetic energies of moving masses, while at low values of the Planck constant $\hbar$ the extent of limitations on quantum transitions is reduced, which manifests itself as an increase in the phase volume in the probability calculations for the processes.

One can also point out the obviously lower contribution from the effects of polarization of the physical vacuum in the course of relativistic motion of matter with respect to the base medium at the early stages of evolution of the dynamics of the Universe, for, according to relations (15), the velocity of light, $c(t)$, at the time had been higher, which had allowed for substantially higher "pre-relativistic" recessional velocities of material objects in accordance with the Hubble law. Thus, the proportion of the component $\left|\varepsilon_{\mathrm{dm}}^{\mathrm{e}}\right|$ in the total energy density $\varepsilon_{\text {tot }}$ had been lower, so that the contribution from the "non-relativistic" baryonic component $\left|\mathcal{E}_{\mathrm{b}}^{\mathrm{e}}\right|$ had been noticeably higher, provided that the quantity $\varepsilon_{\mathrm{V}}^{\mathrm{e}}$ had remained unchanged. This possibility is indirectly confirmed by the inferences that "dark matter" had been absent at the pre-galactic stages of the evolution of the Universe, some 100-200 million years following the Big Bang [34]. Obviously as the velocity $c(t)$ decreases with the age of the Universe in conditions of time common to the entire Universe, the "pre-relativistic" recessional velocities of material objects, including gas-dust components, formed at the early evolution stages and "frozen" in the physical vacuum can become relativistic. This will lead to corresponding changes in the polarization regions of the electromagnetic component of the physical vacuum in the neighborhood of these objects, which governs the relativistic increase of their inertial masses, and hence to the appearance of the $\varepsilon_{\mathrm{dm}}$ component in the total energy density $\varepsilon_{\mathrm{tot}}$. It can be assumed that it was precisely evolutional changes of this type in the dynamics of the Universe that were responsible for the relatively high contribution $(23 \%$ in our epoch, see Sect. 1) of "dark matter" to the total energy balance of the Universe. While the masses originated at the early stages of evolution of the Universe were turning into relativistic ones, there could take place dissipative processes of generation not only of photons, leading to the development of glow owing to the dynamical Casimir effect [26, 27], but also possibly of baryons, because of strong interactions being genetically conditioned by the QED vacuum. In that case, 
there could originate some gas flows forming halos around such galactic clusters. To reveal such halos, provided that they had arisen, it is of interest to conduct investigations into possible differences between the manifestations of "dark matter" at different stages of its formation.

\subsection{Possible Causes of the Anomalies in the Energy Releases of Type Ia Supernovae in Remote Galaxies}

Within the scope of the phenomenological model of the dynamics of the Universe under consideration, one can comprehend possible reasons for the reduction of the energy releases detected on the outbursts of Type I $a$ supernovae in galaxies far away from us (over 4 billion light years) [4-7, 35 ], as compared with the expected ones. Such supernovae are usually considered "standard candles of the Universe", the energy release on their outbursts being quite definite. It were just the anomalies detected which formed the basis for the inferences drawn within the scope of the standard model [4-10] that the expansion of the Universe at red shifts of $z>$ 0.76 , when its size was no more than 0.57 of the size it has in our epoch, took its course with lower velocities than it occurs in our time. Moreover, according to the standard model, later on the expansion velocity had only to grow higher because of the natural reduction of the relative proportion $\varepsilon_{\mathrm{m}}$ of the mass components (see above) against the background of the invariable energy density $\varepsilon_{\mathrm{V}}$ of "black energy" that brings "antigravity" into effect.

To analyze the data available on the energy releases of Type I $a$ supernovae in remote galaxies [11, 12, 35], we consider the relationship between the luminosity distance $D_{\mathrm{L}}(z)$ and the red shift $z$, with due regard for the function $c(H)$ introduced above (see relations $(15)$ ). The quantity $D_{\mathrm{L}}(z)$ is defined as the path traversed by light emitted at the instant $t_{\mathrm{e}}$ by a source located at a distance of $r$ from the coordinate origin $(r=0)$ at which is situated the observer that detects the emitted light at the instant $t_{0}[11,36]$. By definition, the red shift is given by

$$
z=\frac{v_{\mathrm{e}}-v_{0}}{v_{0}}=\frac{a\left(t_{0}\right)}{a\left(t_{\mathrm{e}}\right)}-1
$$

Here $v_{\mathrm{e}}$ and $v_{0}$ are the frequencies of light emitted by the stellar source and received by the observer, respectively, and $a\left(t_{\mathrm{e}}\right)$ and $a\left(t_{0}\right)$ are the "sizes" of the Universe at the instants $t_{\mathrm{e}}$ and $t_{0}$. The distance covered by light, which is determined by the totality of displacements of its wave front along the geodetic line for metric in a homogeneous Euclidean space, with due consideration given for its lengthening by $(1+z)$ times owing to the expansion of the Universe, may be represented, on generalization of the expression presented in [37], in the form

$$
D_{\mathrm{L}}(z)=(1+z) a\left(t_{0}\right) \int_{t_{\mathrm{e}}}^{t_{0}} \frac{c[a(t)] d t}{a(t)}
$$

where relationship is introduced between the velocity of light and the current size of the Universe. Having made the substitution of variable

$$
\xi=\frac{a\left(t_{0}\right)}{a(t)}-1 ; d \xi=-\frac{a\left(t_{0}\right)}{a^{2}(t)} \frac{d a}{d t} d t ; \frac{1}{a(t)} \frac{d a}{d t}=H(\xi)
$$

and a number of transformations, with due regard for relation (15), we get

$$
D_{\mathrm{L}}(z)=(1+z) \int_{0}^{z} \frac{c(\xi)}{H(\xi)} d \xi=\frac{(1+z) c_{0}}{H_{0}^{2 / 3}} \int_{0}^{z} \frac{d \xi}{H^{1 / 3}(\xi)}
$$

In the absence of relation (16), when $c(H)=c_{0}$, the corresponding expression has the form

$$
D_{\mathrm{L}}^{0}(z)=(1+z) c_{0} \int_{0}^{z} \frac{d \xi}{H(\xi)} .
$$

The quantitative analysis of these relationships is complicated by the difficulty of obtaining direct information on the dependence $c(z)$ in distant galaxies, since the integrand in the expression for the luminosity distance contains a combination $c(z) / H(z)$. However, qualitative assessments in this case can be made. Since the function $H=$ $H(\mathrm{z})$ increases with increasing $z$, relationship (18) between the luminosity distance and the red shift proves a more rapidly increasing function than the standard relation $(18 a)$ that is defined at $c(H)=c_{0}$ and actually leads to the underrating of the true distance to the light source cosmological distances away. This means that the phenomenon of reduced energy releases measured for type I $a$ supernovae in distant galaxies, as compared to the standard values in nearby galaxies, is but apparent and can be comprehended without resort to the assumption of accelerated expansion of the Universe in the later epochs, if the hypothesis that the world constants depend on the age of the Universe is adopted.

To conclude this section, let us present one more inference of the phenomenological notions being expounded about the dynamics of the universe, which elucidates the physical meaning of the phenomenological Hubble relation (7). Considering relations (15), the Hubble "radius of the Universe", $R_{\mathrm{H}}(t)$, represented as a limiting form of expression (7), is

$$
R_{\mathrm{H}}(t)=\frac{c(t)}{H(t)}=c_{0} t\left(\frac{H}{H_{0}}\right)^{2 / 3}=c_{0} t_{0}^{2 / 3} t^{1 / 3}
$$

where $t_{0}$ is the age of the Universe in our epoch. This relation means that, given constant $w_{\mathrm{Pl}}$ and $\varepsilon_{\mathrm{tot}}$, the volume $V_{\mathrm{U}}(t)$ of the Universe increases in proportion to time, $V_{\mathrm{U}}(t) \sim R_{\mathrm{H}}{ }^{3} \sim t$, in accordance with relation (5), and so the Hubble law simply reflects the constancy of the average energy density in the expanding Universe being realized with the active Planckian energy-mass source. Note also the reduction of the rate of extension of the "radius of the Universe" with its age, $\dot{R}_{H}(t) \sim t^{-2 / 3}$, in contrast to the inference of the standard model that the expansion of the Universe accelerated in subsequent epochs. 


\section{Physical Vacuum of the Universe and Inflation Vacuum}

The phenomenological notions developed here that the power of the Plnckian energy-mass source is being evenly distributed all over the space of the Universe correspond to Friedmann's equation (8) written down for the case where the entire energy-mass is also assumed to be uniformly distributed over the volume of the Universe. To go over to more adequate models of the dynamics of the Universe that would allow for nonuniformity in the distribution of masses over space, would require introduction of notions about successive origination of local Planckian power sources that integrally maintain the conservation of the fundamental cosmological principle - the homogeneity and isotropism of the distribution of matter over the Universe. Considered as one such possibility may be a temporally consecutive switching-on of Planckian energy-mass sources of chaotically varying localization on the outer sphere of radius $R_{\mathrm{H}}$; i.e., at the boundary between the already formed Universe and the primeval, inflation vacuum whose space had begun being absorbed, following the Big Bang, by the expanding Universe being formed. In other words, we will assume that in contrast to the existing models of the dynamics of the Universe [38, 39] not all of the energy of the inflation vacuum, defined as the "false", metastable vacuum with an energy density exceeding that of the vacuum of the Universe, had been released within a very short time, a tiny fraction of a second following the Big Bang and realized as the observable energy of the Universe. We will take it that the process of such an "assimilation" of the energy of the false vacuum and its concurrent transformation into the energy of the expanding Universe runs incessantly during the course of the fluctuation-induced origination of Planckian power sources in the neighborhood of the boundary between the two vacua, namely, the physical and the false vacuum. It should be assumed at the same time that such a fluctuation arises in the region of the physical vacuum in accordance with the logic of Sect. 2, but cannot be liberated by virtue of the vanishing of the time-like component $g_{00} \sim \psi(r)=-\left(1-r_{S c} / r\right)$ of interval (3), and the release of energy occurs when such a latent excitation comes into contact with the false vacuum. In that case, all of the liberated energy-mass of the Planckian source must be emitted into the region of the existing Universe, this being facilitated by the high "affinity" of the material particles being formed to the QED vacuum, which is characterized by their energy of binding to the latter $[14,16]$. It is quite natural to assume that the entire energy flux of the fluctuation arisen, which is considered as a Planckian energy-mass source, will be localized within a jet - a cone of certain solid angle $\beta_{\mathrm{P} 1}$.

This brings up the natural question: Can a terrestrial observer detect powerful Planckian energy releases continuously taking place in a randomly distributed fashion along the boundary of our Universe? And also how high must be the frequency of such Planckian events for a terrestrial observer to detect them? Obviously the answer to the latter question depends on the characteristic value of the solid angle $\beta_{\mathrm{Pl}}$, which can vary as a function of the evolution dynamics of the fluctuation. The magnitude of the angle $\beta_{\mathrm{Pl}}$ and its variations will govern both the average frequency of detection of such events and the characteristic time it will take for the events being detected to demonstrate with a preset confidence the isotropism of their distribution over the celestial sphere. Let us obtain the upper-bound estimate for the magnitude of the angle $\beta_{\mathrm{Pl}}$ on the assumption that such fluctuation explosions follow one another with equal probability throughout the boundary region between the Universe and the "false" vacuum. We present the condition that the region of a jet with a solid angle of $\beta_{\mathrm{PI}}$ will at least touch an arbitrary region of the Universe during an earthday in the form $\beta_{\mathrm{P} P} R_{\mathrm{H}}{ }^{2} N=\pi R_{\mathrm{H}}^{2}$, where $N$ is the number of Planckian bursts per earthday. Considering that the characteristic duration of each Planckian energy release event is $\tau \approx 100 \mathrm{~s}$ (see below) and $N=864$, we get the following estimate: $\beta_{\mathrm{Pl}} \approx 3.6 \times 10^{-3} \approx 12.5$.

From this standpoint, the only candidate for the Planckian source manifestations being detected are gamma-ray bursters (GRBs), the most powerful and relatively short-lived gamma radiation sources in cosmologically distant regions of the observable Universe [40-42]. These most enigmatic objects in astrophysics, which are being detected once daily on the average, demonstrate isotropic distribution over the celestial sphere. They are characterized by a wide spread of gamma-ray burst durations (from a few milliseconds to a few tens of seconds) and spectral compositions (from $1 \mathrm{keV}$ to $10 \mathrm{MeV}$ ), as well as integral energy releases (from $10^{49}$ to $10^{54} \mathrm{erg}$ ). Sometimes they prove difficult to associate with any galaxy. Frequently one manages to "tie" them in to remote galaxies, thanks to the detection of long-duration excitations in these galaxies, sometimes associated with supernova explosions [43]. At the same time, the probability of detection of supernova explosions accompanied by long-term relaxation fails to correlate with the power of the GRBs. For example, the powerful GRB 060614 [44], even visible by ground-based telescopes, whose lifetime amounted to 102 seconds, was not attended by any supernova explosion. Optical telescopes detected its associated light, 100 times fainter than that of the faintest supernovae. At the same time, the powerful GRB 080 319B caused a strong afterglow of its host galaxy (redshift $z=0.937$ ) [45]. It was the brightest naked-eye object ever seen from our planet. For nearly one minute its brightness was equivalent to the brightness of 10 million typical galaxies. GRB $080319 \mathrm{~B}$ had the true luminosity that was 2.5 million times higher than the one recorded for the most powerful supernova SN 2005ap [46]. The burst was characterized by an energy release of $1.3 \times 10^{54} \mathrm{erg}$ (in terms of isotropic energy release) in the band $1-10^{4} \mathrm{keV}$ [45]. The age of the host galaxy was approximately 7.5 billion years. But no supernova was associated with this gamma-ray burst. The fact that the energy release and luminosity of GRB 080 319B were much higher than the ones recorded for the most powerful supernovae imply that the nature of this 
burst can differ from that of supernovae.

It should be noted that so powerful energy releases in the gamma range associated with gamma-ray bursts were observed earlier as well (see, for example, Ref. No. [47]). Also important to notice is the fact that gamma-ray bursts can occur even in the "darkest" regions of space, where no stars or galaxies exist. The most illustrative example is GRB 070125 [48]. Although various mechanisms of such phenomena (the death of massive stars at the final stage of evolution, mergers of compact objects, e.g., a neutron star and a neutron star or a neutron star and a black hole [40-43]) have been developed to date, the true nature of such objects remains unclear. Of course, the intriguing factor here is the periodicity of occurrence of gamma-ray bursts: one event per earthday. It is hard to imagine a process of fusion involving two stars or a star and a black hole that can occur with such a periodicity in the Universe. It should be borne in mind that the degree of inscrutability of the nature of gamma-ray bursts grows higher because it is customarily believed that the energy released in such celestial processes is concentrated in jets (otherwise it would be difficult to comprehend the physical nature of so powerful energy sources, with the energy issued being isotropic). But since not nearly all of the jets formed can turn out to be oriented on the Solar system, not nearly all of the gamma-ray bursts are being detected. For this reason, the number of such events in the Universe as the fusion of stars must be much greater. But since such large-scale events associated with the fusion of stars and accompanied by powerful gamma-ray bursts have not been detected in relatively near regions of the Universe, one is forced to seek alternative mechanisms underlying the origin of gamma-ray bursts. And certainly the amazing once-a-day periodicity of such large-scale events in the Universe also seems little-probable for the fusion of stellar objects to be realized all over the space of the Universe. From this point of view, the assumption as to the relation between gamma-ray bursts and the fluctuation dynamics of Planckian energy-mass sources in the region of the outer boundary between the Universe and the false vacuum seems to be substantiated. The more so since all the peculiarities of the phenomena that are commonly being associated with gamma-ray bursts, specifically the excitation of galaxies under the effect of the energy fluxes propagating in the form of jets from blinking Planckian energy-mass sources, can be qualitatively comprehended on the basis of this hypothesis. First of all, this is the variation of the duration $\tau_{\mathrm{Pl}}$ of the gamma radiation proper during the course of recording of a gamma-ray burst. The energy fluxes issuing from Planckian energy-mass sources can either cause general excitation in the galaxies getting in the region of the corresponding jets [44] or initiate the "ignition" of supernovae [41-43]. The fluxes of gamma quanta associated with gamma-ray bursts that are being detected by terrestrial observers naturally depend on the orientation of the energy flow in the Universe. To illustrate, it can be supposed that the flux of gamma quanta from the source GRB 08319B [45] was maximally oriented on the Solar system and that the abnormally great duration, $\tau_{\mathrm{Pl}} \sim 100 \mathrm{~s}$, of the gamma signal and the gamma radiation power formally calculated when observing this gamma-ray burst were due exactly to this circumstance. It has been exactly the duration of gamma release from this source that has conditioned the choice of the value for the quantity $\tau_{\mathrm{Pl}}$ when estimating the characteristic value of the solid angle $\beta_{\mathrm{Pl}}$ above. In connection with the hypothesis being discussed as to the relation between gamma-ray bursters and Planckian energy-mass sources, of interest might be observations of excitations of spatially separated fragments of remote galaxies, pointing to influences from extraneous factors, provided that the possibility of cause-effect relations between such excitations are excluded.

To conclude this section, note that the notions being introduced about local releases of the energy of false vacuum in the neighborhood of the interface between the "two vacua", the physical and the false ones, open up new possibilities of considering gamma bursters as possible sources of the cosmic rays with energies over $10^{18} \mathrm{eV}$, whose concomitant flow of energetic neutrinos proves substantially weaker than predicted by the common phenomenological gamma burster models, the "fireball" models in particular. It was exactly such result that was obtained by Abbasi and co-workers [49]. These authors reported that the upper limit to the energetic neutrinos associated with gamma-ray bursts turned out to be lower than predicted by at least a factor of 3.7.

\section{Concluding Remarks}

The approaches suggested in this work to the solution of urgent astrophysical problems are actually a further development of the steady state theory of the expanding Universe [50-53], according to which fresh matter is being constantly created during the course of this expansion. In contrast to the models suggested in the above works, the phenomenological Planckian energy-mass source model under consideration has to do with the generation not only of the baryonic component of matter, but also of the entire energy of the Universe, including "dark energy". Moreover, since it is assumed that the Planckian energy-mass source had originated as the final phase of the inflationary stage of the Big Bang, it had been exactly the action of such a source at the early stages of evolution, characterized by the formation of a homogeneous and superdense Universe with the "hottest" photons, that had to cause generation of the cosmic microwave background ("relict") radiation detected in our epoch, which reflects the history of cooling of the highest-energy "primary" photons [54]. It should be noted that it were precisely the difficulties encountered in analyzing fluctuations of the relict radiation within the scope of the "steady state" model, as well as the impossibility to comprehend the above-discussed differences in specific energy density between the energy sources originated at the early and the subsequent stages of the evolution of the Universe, that were the reason behind the non-acceptance of 
the models [50-53]. It can be assumed that the Plnackian energy-mass source model suggested in this work, which clears up the above-mentioned difficulties, will allow the very idea that energy-mass is being constantly introduced into the evolving Universe to be somewhat "rehabilitated".

From this standpoint, it would be of interest to present quantitative comparisons between the inferences of the given phenomenological model and the results of the latest investigations [55] (see also [56]) into the specific features of "compact" massive galaxies formed at the early stages of the evolution of the Universe, up to 3 billion years following the Big Bang. Wel and co-workers [55] analyzed 14 such compact galaxies. Their sizes proved to be four to five times smaller, and their densities, tens of times higher than those of galaxies containing approximately the same number of stars, but formed in substantially later epochs. The investigators' attention was paid to the cardinal differences in both the dynamics and structure between galaxies of approximately the same mass, but formed in different epochs of the evolution of the Universe. To illustrate, while the velocity of rotation of the Sun about the center of our galaxy is $230 \mathrm{~km} / \mathrm{s}$, the corresponding estimates for the velocity of stars in the "rotating disks" of the compact galaxies yielded $700 \mathrm{~km} / \mathrm{s}$, and while the compact galaxies are disk-shaped, the younger galaxies are predominantly spherical. It would be of interest to perform computer simulations of the processes of formation of stars and galaxies in conditions of continuous production of energy-mass by the Planckian source, with phenomenological consideration being given for the temporal changes of the world constants in different epochs of evolution of the Universe in accordance with relations (15).

It is the author's opinion that the further search for the possibilities of obtaining direct experimental information on the magnitudes of the world constants and those of the various energy density components of the Universe at different values of the red shift $z$ can be considered as one of the cosmological problems of current concern. Such information could have been obtained from direct measurements of the Lamb shift $[57,58]$ in atomic hydrogen spectra in distant galaxies $[56,59,60]$, because the energy difference $\delta E$ between the $2 S_{1 / 2}$ and $2 P_{1 / 2}$ states of the hydrogen atom, due to quantum fluctuations of the electromagnetic component of the physical vacuum, proves dependent, considering relations (15) and (18), on $H$ :

$$
\delta E=0.41 \alpha_{e}^{5} \cdot m_{e} c^{2}=\delta E_{0} \cdot\left(\frac{H_{0}}{H}\right)^{1 / 9},
$$

where $\delta E_{0}$ is the magnitude of the Lamb shift in our epoch (corresponds to a frequency of $1050 \mathrm{MHz}$ ). It should also be noted that the analysis of such effects in galaxies observed at various values of $H=H(z)$ could have also made it possible to find out how much are the above-introduced relations (15) justified.

Recent results Mould and Uddin [61] could be interesting from this point of view. These authors employed the full SNIa dataset to the end of 2013 to set a limit on the Newton's constant $G$ variation. In their approach they adopted the standard candle delineation of the redshift distance relation. They set an upper limit of its rate of change $|\dot{G} / G|$ of 0.1 parts per billion per year over 9 Gyrs, or varG $=90 \%$ over the past 9 billion years. Note, this result is fully consistent with estimates obtained from Eq. (12) of Paper 1 [16] and Eq (15) of this article: $\operatorname{varG}=61 \%$ during past 9 Gyrs. Another possibility to check on the adequacy of the inferences of the phenomenological model of the dynamics of the Universe being developed could have been provided by the analysis of the gravitational redshift of light from clusters of galaxies. This effect differs from the cosmological redshift and is caused exclusively by the expansion of the Universe [62, 63]. Wojtak, Hansen, and Hjorth [62] successfully tested Albert Einstein's gravitation theory on cosmic scales of the order of $1-10 \mathrm{Mpc}$. The study of the gravitational redshift on clusters of distant galaxies, for example, localized at $z \sim 1$ and more, would allow one to ascertain whether or not the world constants vary on cosmological scales.

The objective of any phenomenological approach it is to unite the variety of the experimental information available and represent the interrelations revealed in the form of expressions of definite physical meaning involving physically comprehensible parameters that can be determined experimentally. The phenomenological relationships and parameters being introduced in this work can form the basis for constructing physical models of the various manifestations of the dynamics of the Universe.

\section{References}

[1] Einstein A. Kosmologische Betrachtungen zur allgemeinen Relativitätheorie / Sitzung. Preus. Akad. Wiss. 1917. Bd 1, s. 142-152.

[2] Kant I. Universal Natural History and Theory of the Heavens. University of Michigan Press. 1969, 182 p.

[3] Chaadaev P.Ya. Fourth Philosophical Letter. Library Milestones.

http://www.yabloko.ru/Themes/History/Chaadaev4.html

[4] Perlmutter S., Aldering G., Goldhaber G. et.al. Measurements of $\Omega$ and $\Lambda$ from 42 High-Redshift Supernovae // Astrophys. Journal. 1999. V. 517. P. 565-586.

[5] Schmidt B.P., Suntzeff N.B., Phillips M. M., et al. The High-Z Supernova Search: Measuring Cosmic Deceleration and Global Curvature of the Universe Using Type Ia Supernovae // Astrophys. J. 1998. V. 507. p. 46-63.

[6] Riess A., Filippenko A., Challis P. et al. Observational Evidence from Supernovae for an Accelerating Universe and a Cosmological Constant // Astron. J. 1998. V. 116. P. 1009-1038. arXiv:astro-ph/9805201

[7] Perlmutter S. Supernovae, Dark Energy, and the Accelerating Universe. Physics Today. 2003. V. 56. N 4. P. 53-60.

[8] Rubakov V.A. Hierarchies of fundamental constants (to items Nos 16, 17, and 27 from Ginzburg's list) // Physics - Uspekhi. Advances in Physical Sciences. 2007. V. 50. N 4. P. 390-396. 
[9] Sahni V. Dark matter and dark energy // Lect. Notes Phys. 2004. V. 653. P. 141-180; arxiv.org/abs/astro-ph/0403324v3.

[10] Chernin A.D. Dark energy and universal antigravitation // Physics - Uspekhi. Advances in Physical Sciences. 2008. V. 51. N 3. P. 253-282.

[11] Padmanabhan T. Darker side of the Universe. 29 International Cosmic Ray Conference Pune. 2005. V. 10. P. $47-62$.

[12] T. Reichhardt. Cosmologists look forward to clear picture // Nature. 2003. V. 421. P. 777.

[13] Zel'dovich Ya.B. Vacuum theory: a possible solution to the singularity problem of cosmology // Physics - Uspekhi. Advances in Physical Sciences. 1981. V. 24. N 3. P. 216-230.

[14] Timashev S.F. Preprint: Physical vaccum as a system manifesting itself on various scales - from nuclear physics to cosmology: http://arxiv.org/abs/1107.1799v7.

[15] Burlankov D.E. Time, space, gravity. Moscow-Izhevsk: NIC "Regular and Chaotic Dynamics". 2006. 420 p. (in Russian).

[16] Timashev S.F. // Dynamic essence of the basic relations of the special relativity theory and the origin of the fundamental interactions: phenomenology // Paper 1.

[17] Greene B. The Elegant Universe. Vintage Books. A Division of Random House, Inc. New York. 1999.

[18] Klapdor-Kleingrothaus H.V., Zuber K. Teilchenastrophysik. B.G. Teubner GmbH, Stuttgart, 1997.

[19] Lanczos C. The Variational Principles of Mechanics. Toronto: Univ. Toronto Press, 1964.

[20] Feynman R.P., Leighton R.B., Sands M. The Feynman Lectures on Physics. Vol. 1: Mechanics. Heat. Radiation. Chap. 15, § 9. Addison Wesley Reading Mass. 1966.

[21] Spergel D. N.; Verde L., Peiris H.V. et al. First Year Wilkinson Microwave Anisotropy Probe (WMAP) Observations: Determination of Cosmological Parameters // The Astrophysical Journal Supplement Series. 2003. V. 148. P. 175-194.; arXiv:astro-ph/0302209.

[22] Perelman G. The entropy formula for the Ricci flow and its geometric applications. http://arXiv:math/0211159v1.

[23] Brooks D.W.C., Botter T, Schreppler S. Non-clqssic light generated by quantum-noise-driven cavity optomechanics // Nature. 2012. V.488. P. 476-480.

[24] Klimchitskaya G.L., Mohideen U., Mostepanenko V.M. The Casimir force between real materials: experiment and theory // Rev Mod. Phys. 2009. V. 81. P. 1827-1885.

[25] Mostepanenko V.M., Trunov N.N. The Casimir effect and its applications // Physics - Uspekhi. Advances in Physical Sciences. 1988. V. 31. N 3. P. 965-987.

[26] Dalvit D.A.R., Neto P.A.M., Mazzitelli F.D. Fluctuations, dissipation and the dynamical Casimir effect. http://arxiv.org/abs/1006.4790v2.

[27] Wilson C.M., Johansson G., Pourkabirian A., Johansson J.R., Duty T., Nori F., Delsing P. Observation of the Dynamical Casimir Effect in a Superconducting Circuit. http://arxiv.org/pdf/1105.4714v1.
[28] Weinberg S. Cosmology. Oxford: University Press. 2008, 593 p.

[29] Okun' L.B. Physics of elementary particles. Moscow: Nauka. 1984, 224 p. (in Russian).

[30] Chand H., Srianand R., Petitjean, Aracil B. Probing the cosmological variation of the fine structure constant: Results based on the VLT-UVES sample; http://arXiv:astro-ph/0401094v1.

[31] Zel'dovich Ya.B., Novikov I.D. The Structure and Evolution of the Universe. Chicago: University of Chicago, 1983.

[32] Delvin M.J., Ade P.A.R., Aretxaga I., et al. Over half of the far-infrared background light comes from galaxies at $\mathrm{z} \geq 1.2$ // Nature. 2009. V.458. P. 737-739.

[33] Nagao T., Maiolino R., Breuck C. De et al. ALMA reveals a chemically evolved submillimeter galaxy at $\mathrm{z}=4.76 / /$ Astronomy and Astrophysics. 2012. V. 542. L34-38; arXiv:1205.4834v2 [astro-ph.CO] 29 May 2012.

[34] Hogan C.J. Ripples of early starlight // Nature. 2007. V. 445. P. 37.

[35] Amanullah R., Lidman C., Rubin D., et al. Spectra and Hubble Space Telescope Light Curves of Six Type Ia Supernovae at $0.511<z<1.12$ and the Union2 Compilation // The Astrophysical Journal, Volume 716, Issue 1, pp. $712-738$ (2010).

[36] Plebański J., Krasiński A. An introduction to General Relativity and cosmology. Cambridge: Cambridge University Press. 2006, 534 p.

[37] Starobinsky A.A. How to determine an effective potential for a variable cosmological term // JETP Lett. 1998. V. 68, No. 9-10. P. 721-726.

[38] Vilenkin A. Many worlds in one: The search for other universes. New York: Hill and Wang. 2006, 235 p.

[39] Linde A. Inflation, Quantum Cosmology and the Anthropic Principle. In: "Science and Ultimate Reality: From Quantum to Cosmos", honoring John Wheeler's 90th birthday. J. D. Barrow, P.C.W. Davies, \& C.L. Harper eds. Cambridge: Cambridge University Press, 2003.

[40] Pen Ue-Li, Loeb A. Gamma-ray bursts from barion decay in neutron stars. // The Astrophys. Journal. 1998. V.509. P.537-543.

[41] Marshal H.L. The evidence in the afterglow // Nature. 2002. V.416. P.484-485.

[42] Plaga R. Rays from the dark. Nature. 2008. V. 453. P. 48-49.

[43] Reeves J.N., Watson D., Osborne J.P. et al. The signature of supernova ejecta in the ray afterglow of the $\gamma$-ray burst 011211. // Nature. 2002. V.416. P.512-515.

[44] Gehrels N., J. P. Norris J. P., Barthelmy J. P. et al. A new $\gamma$-ray burst classification scheme from GRB 060614. Nature. 2006. V. 444. P. 1044-1046.

[45] Bloom J. S., Perley D. A., Li W. Observations of the Naked-Eye GRB 080319B: Implications of Nature's Brightest Explosion. The Astrophysical Journal. 2009. V. 691. N 1. P. 723-737. 
[46] Quimby R.M., Aldering G, Wheeler J.C. et al. SN 2005ap: A Most Brilliant Explosion. http://arXiv:astro-ph/0709.0302v1 (Sep 2007).

[47] Blandford R.D., Helfand D.J. Will GRB 990123 Perform an Encore? http://arXiv:astro-ph/9902004v2 (Feb 1999).

[48] Cenko, S.B., et al. GRB 070125: The first long-duration gamma-ray burst in a halo environment // Astrophys. J. 2008. V. 677. P. 441- 447.

[49] Abbasi R., Abdou Y., Abu-Zayyad T. et al. An absence of neutrinos associated with cosmic-ray acceleration in $\gamma$-ray bursts // Nature. 2012. V. 484. P. 351-354].

[50] Bondi H., Gold T. The Steady-State Theory of the Expanding Universe // Monthly Notices Roy. Astron. Soc. 1948. V. 108. P. $252-270$.

[51] Hoyle F. New model for the expanding Universe // Monthly Notices Royal Astron. Soc. 1948. V. 108. P. 372-382.

[52] Hoyle F., Burbidge G., Narlikar J.V. A quasi-steady state cosmological model with creation of matter // The Astrophysical Journal. 1993. V. 410. P. 437-457.

[53] Hobson M.P., Efstathiou G.; Lasenby A.N. General Relativity: An Introduction for Physicists. Cambridge University Press, 2006. $388 \mathrm{p}$.

[54] Massey R. Dark is the new black. Nature. 2009. V. 461. P. 740-741.
[55] Wel A. van der, Rix H.-W., Wuyts S., et al. The majority of compact massive galaxies at $z \sim 2$ are disk dominated // Astrophys. J. 2011. V. 730. P. 38-41.

[56] Dokkum Pieter van. Era of the compact disk // Nature. 2011. V. 473. P. 160-161.

[57] G.W. Erickson. Improved Lamb-shift calculation for all values of Z // Phys. Rev. Lett. 1971. V. 27. P. 780-783.

[58] Akhiezer A.I., Berestetskii. Quantum electrodynamics. Moscow: Nauka. 1981. 428 p. (in Russian).

[59] Bouwens R.J., Illingworth G.D., Labble I., Oesch P.A. et al. A candidate redshift $z \approx 10$ galaxy and rapid changes in that population at an age of $500 \mathrm{Myr} / /$ Nature. 2011. V. 469. P.504-507.

[60] Robertson B.E., Ellis R.S., Dunlop J.S., McLure R.J., Stark D.P. Early star-forming galaxies and the reionization of the Universe // Nature. 2010. V. 468. P. 49-55.

[61] Mould J., Uddin S.A. Constraining a possible variation of G with Type Ia Supernovae; Arxiv:1402.1534v2 [astro-ph.CO].

[62] Wojtak W., Hansen S.H., Hjorth J. Gravitational redshift of galaxies in clusters as predicted by general relativity. Nature. 2011. V. 477. P. 567-569.

[63] Wegner G. Gravity tested on large scales. Nature. 2011. V. 477. P. 541-543. 